\title{
SUSCEPTIBILITY TO ORAL SQUAMOUS CELL CARCINOMA: CORRELATION WITH VARIANTS OF CYP1A1-MsPI, GSTT1, GSTM1, ALDH2, EC-SOD AND LIFESTYLE FACTORS
}

\author{
Dong T-T*, Wang L-J, Liu L-Z, Ma S-N
}

\begin{abstract}
*Corresponding Author: Mrs. Ting-Ting Dong, General Hospital of Daqing Oil Field, No. 9 Zhongkang Street, Saertu District, Daqing 163001, Heilongjiang Province, People's Republic of China. Tel: +86-459-599-4114. Fax: +86-459-580-5247. Email: tingtingdong_139@163.com
\end{abstract}

\section{ABSTRACT}

In order to investigate the association between polymorphisms in genes encoding metabolizing enzymes ( $C Y$ P1A1-MspI, EC-SOD (extracellular superoxide dismutase), GSTT1, GSTM1, ALDH2), cigarette and alcohol consumption, and the risk of oral squamous cell carcinoma, we conducted a prospective case-control study comprised of 750 individuals with oral squamous cell carcinoma (OSCC) and 750 healthy individuals. Data about smoking and drinking habits were collected along with other demographic and clinical information. Peripheral blood samples were collected for DNA extraction, and polymerase chain reaction (PCR) and PCR-RFLP (restriction fragment length polymorphism) were used to determine genotypes of CYP1A1, EC-SOD, GSTT1, GSTM1, ALDH2. The results showed that smoking and alcohol consumption were significantly more common among patients than controls $(p<0.05)$. There were significant differences in the genotype distribution for each locus between groups, with the CYP1A1 (m2/ $\mathrm{m} 2), E C-S O D(\mathrm{C} / \mathrm{G})$, GSTT1 [-], GSTM1 [-] and ALDH2 (non $\mathrm{G} / \mathrm{G}$ ) genotypes being more common among patients $(p<0.05)$. Furthermore, the majority of patients had at least two or more variant genotypes, while controls had one or no variant genotype $(p<0.05)$. Finally, multiple variant genotypes combined with smoking, drinking, or both smoking and drinking significantly increased the risk of OSCC, with greater increase for heavier smoking/drinking. In brief, genetic polymorphism of CYP1A1, EC-SOD, GSTT1, GSTM1, and ALDH2 and smoking and drinking history are closely associated with susceptibility to OSCC.

Keywords: Drinking; genetic polymorphism; oral squamous cell carcinoma (OSCC); smoking.

\footnotetext{
General Hospital of Daqing Oil Field, Daqing 163001, Heilongjiang Province, China
}

\section{INTRODUCTION}

Head and neck squamous cell carcinoma (HNSCC) is a common malignancy that ranks sixth in incidence of all cancers. The HNSCC tumors display dysregulation of cell differentiation, cell cycle control, epithelial and stromal interactions, apoptosis, angiogenesis and their associated pathways $[1,2]$. Although its exact cause remains unclear, like most malignancies, HNSCC pathogenesis is affected by both genetic and environmental factors [1].

Of the approximately 500,000 new cases of HNSCC each year, many occur in the oral cavity, pharynx, and larynx. Oral squamous cell carcinoma (OSCC) is the most common type of HNSCC, and China has one of the highest incidences of this cancer [2]. Importantly, OSCC is nearly asymptomatic, which makes early diagnosis very difficult; to date, there are no accurate predictors of OSCC onset and/or progression. Therefore, identification of risk factors and high-risk populations for OSCC would enable advancements in the primary and secondary prevention of OSCC.

Cigarette smoking and alcohol consumption are known environmental risk factors for OSCC $[3,4]$. Cigarette smoke contains polycyclic aromatic hydrocarbons, heterocyclic amines, and nitrosamines that are all carcinogenic. Long-term alcohol consumption can lead to combined overdose of reactive oxygen species (ROS) and acetaldehyde, inducing carcinogenesis. Certain enzymes have been shown to be responsible for the biotransformation of chemical carcinogens, either for activation or excretion. For example, cytochrome P4501A1, encoded by CYP1A1, is a catabolite-activating enzyme involved in the biotransformation of both tobacco and alcohol. P4501 A1mediated metabolism of tobacco combustion products, mainly polycyclic aromatic hydrocarbons, can lead to 
the formation of DNA adducts that contribute to tumor formation, specifically HNSCC [5]. Other metabolizing enzymes, with variations in their respective genes, have also been previously implicated in cancer susceptibility. These include, but are not limited to, glutathione S-transferase (GST), superoxide dismutate (SOD), proteins of the SOD family, and acetaldehyde dehydrogenase (ALDH). Glutathione S-transferase plays a role in metabolizing benzo[a]pyrene (a tobacco-specific carcinogen), as well as other carcinogenic compounds. Superoxide dismutase, an endogenous antioxidant enzyme, has certain polymorphisms implicated in cancer susceptibility. Acetaldehyde dehydrogenase, along with alcohol dehydrogenase (ADH), metabolizes ethanol by breaking apart the molecule in order to eliminate it from the body. Genetic polymorphism in ALDH2 has been previously investigated and shown to be associated with specific cancer types [5]. However, genes encoding these enzymes have multiple functional variants, blurring their role in OSCC susceptibility. At least one recent study found that expression of CYP1A1 and ALDH2 proteins did not affect OSCC prognosis [6]. Thus, the specific contribution of polymorphisms in genes encoding enzymes involved in biotransformation of alcohol and tobacco components remains unclear; specifically, the role in promoting OSCC requires further study. This study reports the investigation of the association between genetic polymorphism of CYP1A1, EC-SOD (extracellular SOD), GSTT1, GSTM1, ALDH2, smoking and alcohol consumption, and susceptibility to oral squamous cell carcinoma.

\section{PARTICIPANTS AND METHODS}

Participants. This prospective study included 750 patients who were admitted to our hospital from June 2011 to May 2015. Another 750 participants who received physical examinations during the same time period were selected as healthy controls; the physical examination showed no cancer or hereditary diseases. There was no statistically significant difference in age, gender, place of origin or nationality, and the subjects were unrelated. Participant demographic data, smoking history, alcohol drinking history, occupational history and family tumor history were collected. The smoking status was evaluated using the smoking index (SI) that was the product of the daily number of cigarettes multiplied by number of years of smoking. Based on SI values, the participants were divided into the following categories: non smokers, individuals with $\mathrm{SI} \leq 400$, and individuals with SI 400. Alcohol consumption was evaluated with the drinking index (DI) that was the product of the daily amount of drinking (in grams) multiplied by the number of years of drinking. Based on DI values, the participants were classified as non drinkers, those with DI $\leq 3000$, and those with DI 3000. The study was approved by the General Hospital of Daqing Oil Field, Daqing, Heilongjiang Province, People's Republic of China (PRC). Informed written consent was obtained from all participants.

Genotyping. From each participant, $3 \mathrm{~mL}$ blood was collected in vacutainers with EDTA as anticoagulant. The QIAmpDNA extraction kit (Qiagen GmbH, Hilden, Germany) was used to extract DNA from white blood cells. Extracted DNA was stored at $-30{ }^{\circ} \mathrm{C}$. Polymerase chain reaction (PCR) was used to amplify the DNA to the levels required for restriction fragment length polymorphism (RFLP) analysis. For all reactions, a total volume of 25 $\mu \mathrm{L}$ comprised $2.5 \mu \mathrm{L} 10 \times$ buffer, $2.5 \mu \mathrm{L}$ dNTP, 20 pmol upstream primers, 20 pmol downstream primers (see below), $0.75 \mu \mathrm{L}$ Taq DNA polymerase (all PCR reagents from Promega, Madison, WI, USA), and 100 ng template DNA. Reactions were performed on PE480 thermocycler (Perkin Elmer?, Norwalk, CT, USA), as follows: initial denaturation at $94{ }^{\circ} \mathrm{C}$ for 4 min., and 35 cycles of denaturation at $94{ }^{\circ} \mathrm{C}$ for 40 seconds, annealing at $55^{\circ} \mathrm{C}$ for 40 seconds, and extension at $72{ }^{\circ} \mathrm{C}$ for 50 seconds, and final extension at $72{ }^{\circ} \mathrm{C}$ for $5 \mathrm{~min}$. The PCR products were digested with restriction endonucleases as appropriate (described below). A total reaction volume of $20 \mu \mathrm{L}$ comprised $1 \mathrm{ng}$ PCR product, $2 \mu \mathrm{L} 10 \times$ NEB (New England Biolabs, Ipswich, MA, USA) reaction buffer, and $10 \mathrm{U}$ endonuclease; reactions were performed at $37^{\circ} \mathrm{C}$ for 3 hours. Digestion products were separated by $100 \mathrm{~V}$ electrophoresis on a $3.0 \%$ agarose gel, for 1 hour. After $30 \mathrm{~min}$. in ethidium bromide, bands were detected by ultraviolet light.

The CYP1A1-MspI polymorphism was detected using the following primer sequences: upstream primer (5'-CAG TGAAGA GGT GTA GCC GCT-3'), downstream primer (5'-TAG GAG TCT TGT CTC ATG CCT-3' (synthesized by TaKaRa, Dalian, China). Restriction digestion with $M s p I$ produced three genotypes: wild-type $(\mathrm{m} 1 / \mathrm{m} 1)$ with a band at $340 \mathrm{bp}$, heterozygous $(\mathrm{m} 1 / \mathrm{m} 2)$ with bands at 340, 200 and $140 \mathrm{bp}$, and homozygous mutant (m2/m2) with bands at 200 and $140 \mathrm{bp}$.

The EC-SOD polymorphism was detected using primer sequences as reported in a previous study [7]: upstream primer (5'-GCA ACC AGG CCA GCG TGG AGA ACG GGA A-3'), and downstream primer 5'-CCA GAG GAG AAG CTC AAA GGC AGA-3'). The PCR product was digested with restriction endonuclease PauI. Two genotypes were produced: homozygous $\mathrm{C} / \mathrm{C}$ with bands 
at 111 and $109 \mathrm{bp}$; and heterozygous $\mathrm{C} / \mathrm{G}$ with bands at 220, 111 and 109 bp.

The GSTT1 polymorphism was detected using primer sequences according to Wilson et al. [8]: upstream primer (5'-TCT CCT TAC TGG TCC TCA CAT CTC-3'), and downstream primer ( 5 '-TCA CCG GAT CAT GGC CAG CA-3'). GSTT1 positive [+] was indicated by the presence of a $480 \mathrm{bp}$ fragment after PCR amplification; GSTT1 negative [-] meant lack of $\mathrm{PCR}$ product. The control $\beta$-globin gene was detected with upstream primer (5'-CAA GAG CCA ACC ACA GGT AC-3') and downstream primer (5'-GAA GAG CCA AGG ACA GGT AC-3').

The GSTM1 polymorphism was detected using primer sequences selected according to Nguyen et al. [9]: upstream primer (5'-GAA CTC CCT GAAAAG CTAAAG C-3'), and downstream primer (5'-GTT GGG CTC AAA TAT ACG GTG G-3'). GSTM1 positive [+] indicated the presence of bands at 230 and $219 \mathrm{bp}$; GSTM1 negative [-] indicated the presence of only one $219 \mathrm{bp}$ band. The control $\beta$-globin gene was detected with upstream primer (5'CAA GAG CCAACC ACA GGT AC-3'), and downstream primer (5'-GAA GAG CCA AGG ACA GGT AC-3').

The $A L D H 2$ polymorphism was detected using primer sequences selected according to Ishibashi et al. [10]: upstream primer ( $5^{\prime}$-CCC TTT GGT GGC TAG AAG ATG-3'), and downstream primer (5'-CCA CAC TCA CAG TTT TCT CTT-3'). The PCR products were digested with the restriction endonuclease $M b o I$. The amplified fragment was $91 \mathrm{bp}$ and three genotypes could be seen after digestion: homozygous $\mathrm{G} / \mathrm{G}$ with a band at $55 \mathrm{bp}$, heterozygous $\mathrm{G} / \mathrm{L}$ with bands at 65 and $55 \mathrm{bp}$, and homozygous L/L with a band at 65 bp. The ALDH2 (G/L) and $\mathrm{ALDH} 2(\mathrm{~L} / \mathrm{L}$ ) were combined and marked as ALDH2 (non $\mathrm{G} / \mathrm{G})$ for co-analysis.
Statistical Methods. The difference in genotype distributions between the patient and control groups was determined using the $\chi^{2}$ test. A non conditional logistic regression model was used to analyze the adjusted odds ratio (OR) for risk of oral squamous cell carcinoma with different genotypes, as well as the combined effects between smoking, drinking, and genotype. The 95\% confidence interval (CI) is also reported. The Statistical Package for the Social Sciences (SPSS Inc., Chicago, IL, USA) version 11.0 was used for statistical analysis. Statistical significance was accepted at a $p$ value of $<0.05$.

\section{RESULTS}

Oral Squamous Cell Carcinoma and Lifestyle. General demographic and clinical information for participants in both groups are provided in Table 1. There was no statistically significant difference in gender or age between case and control groups $(p>0.05)$. However, smoking and drinking were significantly more common in individuals with OSCC than in controls $(p<0.01)$.

Genotype Distribution. For each of the enzyme encoding genes we investigated, the distribution of genotypes, significantly differed between cases and controls (all $p<0.01)$. CYP1A1-MspI (m2/m2), EC-SOD (C/G), GSTT1 [-], GSTM1 [-] and ALDH2 (non G/G) genotypes were significantly more common in individuals with OSCC than in control individuals $(p<0.01)$ (Table 2$)$.

Multiple Genotype Variants Distribution. Because there was a significant shift of the genotype frequencies observed in individuals with OSCC compared to controls, we also examined whether OSCC patients were more likely to carry multiple variant genotypes than healthy subjects. The combined distribution of CYP1A1-MspI (m2/ m2), EC-

Table 1. General characteristics of healthy controls and patients with oral squamous cell carcinoma.

\begin{tabular}{|c|c|c|c|c|c|c|}
\hline Characteristics & & $\begin{array}{c}\text { Control Group } \\
(n=750)\end{array}$ & $\begin{array}{l}\text { Patient Group } \\
\quad(n=750)\end{array}$ & OR Value & $95 \%$ CI & $p$ Value \\
\hline $\operatorname{Sex} n(\%)$ & $\begin{array}{l}\text { males } \\
\text { females }\end{array}$ & $\begin{array}{l}498(66.40 \%) \\
252(33.60 \%)\end{array}$ & $\begin{array}{l}504(67.20 \%) \\
246(32.80 \%)\end{array}$ & 0.97 & $0.78-1.20$ & $>0.05$ \\
\hline Age & years; mean \pm SD & $55.51 \pm 4.42$ & $55.49 \pm 4.33$ & - & - & $>0.05$ \\
\hline $\begin{array}{l}\text { Smoking status } \\
n(\%)\end{array}$ & $\begin{array}{l}\text { non-smoker } \\
\text { smoker } \\
\text { SI } \leq 400 \\
\text { SI } 400\end{array}$ & $\begin{array}{r}451(60.13 \%) \\
299(39.87 \%) \\
202(26.93 \%) \\
97(12.93 \%) \\
\end{array}$ & $\begin{array}{l}265(35.33 \%) \\
485(64.67 \%) \\
120(16.00 \%) \\
365(48.67 \%) \\
\end{array}$ & $\begin{array}{l}2.76 \\
6.33\end{array}$ & $\begin{array}{l}2.24-3.40 \\
4.61-8.71\end{array}$ & $\begin{array}{l}<0.01 \\
<0.01\end{array}$ \\
\hline $\begin{array}{l}\text { Drinking status } \\
n(\%)\end{array}$ & $\begin{array}{l}\text { did not drink } \\
\text { did drink alcohol } \\
\text { DI } \leq 3000 \\
\text { DI } 3000\end{array}$ & $\begin{array}{l}468(62.40 \%) \\
282(37.60 \%) \\
172(22.93 \%) \\
110(14.67 \%)\end{array}$ & $\begin{array}{l}256(34.13 \%) \\
494(65.87 \%) \\
114(15.20 \%) \\
380(50.67 \%)\end{array}$ & 3.20 & $\begin{array}{l}2.59-3.96 \\
5.79-7.16\end{array}$ & $\begin{array}{l}<0.01 \\
<0.01\end{array}$ \\
\hline
\end{tabular}

OR: odds ratio; 95\% CI: 95\% confidence interval; SD: standard deviation; SI: smoking index; DI: drinking index. 
Table 2. Distribution of CYP1A1-MspI, EC-SOD, GSTT1, GSTM1, and ALHD2 genotypes in the control and patient groups.

\begin{tabular}{|c|c|c|c|c|c|c|c|}
\hline \multirow[t]{2}{*}{ Genotype } & \multicolumn{2}{|c|}{ Control Group } & \multicolumn{2}{|c|}{ Patient Group } & \multirow{2}{*}{ OR Value } & \multirow[t]{2}{*}{$95 \%$ CI } & \multirow[t]{2}{*}{$p$ Value } \\
\hline & $n$ & $\%$ & $n$ & $\%$ & & & \\
\hline $\begin{array}{l}\text { CYP1A1-MspI: } \\
\mathrm{m} 1 / \mathrm{m} 1+\mathrm{m} 1 / \mathrm{m} 2 \\
\mathrm{~m} 2 / \mathrm{m} 2\end{array}$ & $\begin{array}{l}593 \\
157\end{array}$ & $\begin{array}{l}79.00 \\
20.93\end{array}$ & $\begin{array}{l}463 \\
287\end{array}$ & $\begin{array}{l}61.73 \\
38.37\end{array}$ & $\begin{array}{l}1.00 \\
2.34\end{array}$ & $1.86-2.95$ & $<0.01$ \\
\hline $\begin{array}{l}\text { EC-SOD: } \\
\mathrm{C} / \mathrm{C} \\
\mathrm{C} / \mathrm{G}\end{array}$ & $\begin{array}{l}605 \\
145\end{array}$ & $\begin{array}{l}80.67 \\
19.33\end{array}$ & $\begin{array}{l}380 \\
370\end{array}$ & $\begin{array}{l}50.67 \\
49.27\end{array}$ & $\begin{array}{l}1.00 \\
4.06\end{array}$ & $3.23-5.12$ & $<0.01$ \\
\hline $\begin{array}{l}\text { GSTT1: } \\
{[+]} \\
{[-]} \\
\end{array}$ & $\begin{array}{l}598 \\
152 \\
\end{array}$ & $\begin{array}{l}79.73 \\
20.27\end{array}$ & $\begin{array}{l}395 \\
355 \\
\end{array}$ & $\begin{array}{l}52.67 \\
47.33\end{array}$ & $\begin{array}{l}1.00 \\
3.54\end{array}$ & $2.81-43.44$ & $<0.01$ \\
\hline $\begin{array}{l}\text { GSTM1: } \\
{[+]} \\
{[-]}\end{array}$ & $\begin{array}{l}419 \\
331\end{array}$ & $\begin{array}{l}55.87 \\
44.13\end{array}$ & $\begin{array}{l}231 \\
519\end{array}$ & $\begin{array}{l}30.80 \\
69.20\end{array}$ & $\begin{array}{l}1.00 \\
2.83\end{array}$ & $1.95-4.47$ & $<0.01$ \\
\hline $\begin{array}{l}\text { ALHD2: } \\
\mathrm{G} / \mathrm{G} \\
\text { non } \mathrm{G} / \mathrm{G}\end{array}$ & $\begin{array}{l}444 \\
306\end{array}$ & $\begin{array}{l}55.60 \\
44.40\end{array}$ & $\begin{array}{l}212 \\
538\end{array}$ & $\begin{array}{l}30.53 \\
69.47\end{array}$ & $\begin{array}{l}1.00 \\
3.68\end{array}$ & $2.97-4.57$ & $<0.01$ \\
\hline
\end{tabular}

OR: odds ratio; $95 \%$ CI: 95\% confidence interval.

Table 3. The combined distribution of CYP1A1-MspI (m2/m2), EC-SOD (C/G), GSTT1[-], GSTM1 [-] and ALHD2 (non G/G) variant genotypes in the control and patient groups.

\begin{tabular}{|c|c|c|c|c|c|c|c|}
\hline \multirow{2}{*}{$\begin{array}{c}\text { Number of } \\
\text { Genotypes }\end{array}$} & \multicolumn{2}{|c|}{ Control Group } & \multicolumn{2}{|c|}{ Patient Group } & OR Value & 95\% CI & $p$ Value \\
\cline { 2 - 8 } & $n$ & $\%$ & $n$ & $\%$ & & & \\
\hline 0 & 97 & 12.93 & 8 & 1.07 & 1.00 & - & $<0.01$ \\
\hline 1 & 290 & 38.67 & 64 & 8.53 & 2.68 & $1.23-5.78$ & - \\
\hline 2 & 210 & 28.00 & 230 & 30.67 & 13.28 & $6.31-27.97$ & - \\
\hline 3 & 90 & 12.00 & 278 & 37.07 & 37.45 & $17.53-80.02$ & - \\
\hline 4 & 56 & 7.47 & 156 & 20.80 & 33.78 & $15.44-73.90$ & - \\
\hline 5 & 7 & 0.93 & 14 & 1.86 & 24.25 & $7.61-77.27$ & - \\
\hline
\end{tabular}

OR: odds ratio; $95 \%$ CI: $95 \%$ confidence interval.

Table 4. Combined analysis of smoking status, and variant genotype distribution in relation to oral squamous cell carcinoma susceptibility.

\begin{tabular}{|c|c|c|c|c|c|c|c|c|}
\hline \multirow{2}{*}{$\begin{array}{c}\text { Number of } \\
\text { Genotypes }\end{array}$} & \multirow{2}{*}{$\begin{array}{c}\text { Smoking } \\
\text { Status }\end{array}$} & \multicolumn{2}{|c|}{ Control Group } & \multicolumn{2}{|c|}{ Patient Group } & OR Value & 95\% CI & $p$ Value \\
\cline { 3 - 9 } & $n$ & $\%$ & $n$ & $\%$ & & & \\
\hline 0 & {$[-]$} & 92 & 12.27 & 31 & 4.13 & 1.00 & - & - \\
\hline 1 & {$[-]$} & 118 & 15.73 & 43 & 5.73 & 1.04 & $0.64-1.70$ & 0.86 \\
\hline 2 & {$[-]$} & 116 & 15.47 & 90 & 12.00 & 2.22 & $1.44-3.44$ & $<0.01$ \\
\hline 3 & {$[-]$} & 71 & 9.47 & 53 & 7.07 & 2.14 & $1.31-3.50$ & $<0.01$ \\
\hline 4 & {$[-]$} & 40 & 5.33 & 46 & 6.13 & 3.30 & $1.92-5.67$ & $<0.01$ \\
\hline 5 & {$[-]$} & 14 & 1.87 & 2 & 0.27 & 0.41 & $0.09-1.87$ & 0.25 \\
\hline 0 & {$[+]$} & 37 & 4.93 & 14 & 1.87 & 0.89 & $0.43-1.86$ & 0.76 \\
\hline 1 & {$[+]$} & 100 & 13.33 & 92 & 12.27 & 2.52 & $1.61-3.96$ & $<0.01$ \\
\hline 2 & {$[+]$} & 78 & 10.40 & 147 & 19.60 & 2.43 & $1.65-3.58$ & $<0.01$ \\
\hline 3 & {$[+]$} & 35 & 4.67 & 128 & 17.07 & 4.90 & $2.92-8.21$ & $<0.01$ \\
\hline 4 & {$[+]$} & 44 & 5.87 & 81 & 10.80 & 1.60 & $0.91-2.80$ & 0.10 \\
\hline 5 & {$[+]$} & 5 & 0.67 & 23 & 3.07 & 32.20 & $5.49-118-91$ & $<0.01$ \\
\hline
\end{tabular}

OR: odds ratio; $95 \%$ CI: 95\% confidence interval. 
SOD (C/G), GSTT1 [-], GSTM1 [-] and ALHD2 (non G/G) genotypes in both groups are provided in Table 3. Within the patient group, the majority of individuals (90.4\%) had at least two of the variants. Namely, $1.9 \%$ of individuals were positive for five of the variant genotypes, $20.8 \%$ for four genotypes, $37.1 \%$ for three genotypes, $30.7 \%$ for two genotypes, $8.5 \%$ for only one variant genotype, and only $1.1 \%$ did not have any of the variant genotypes. In contrast, in the control group, the majority of individuals had one or no variants $(51.6 \%)$. Thus, the presence of two or more variants in the OSCC population was significantly more common than in controls $(p<0.01)$.

Smoking Status and Gene Polymorphisms. Table 4 depicts the results of the analysis of CYP1A1-MspI (m2/ $\mathrm{m} 2), E C-S O D(\mathrm{C} / \mathrm{G})$, GSTT1 [-], GSTM1 [-] and ALHD2 (non $\mathrm{G} / \mathrm{G}$ ) variant genotype presence and smoking status. In individuals with OSCC, multiple variants and smoking were significantly more common compared to the control group; smoking in combination with multiple variant genotypes had a synergistic effect on cancer likelihood.

Table 5. Combined analysis of smoking index, and variant genotype distribution in relation to oral squamous cell carcinoma susceptibility.

\begin{tabular}{|c|c|c|c|c|c|c|c|c|}
\hline \multirow{2}{*}{$\begin{array}{c}\text { Number of } \\
\text { Genotypes }\end{array}$} & \multirow{2}{*}{$\begin{array}{c}\text { Smoking } \\
\text { Status }\end{array}$} & \multicolumn{2}{|c|}{ Control Group } & \multicolumn{2}{|c|}{ Patient Group } & OR Value & 95\% CI & $p$ Value \\
\cline { 3 - 9 } & $n$ & $\%$ & $n$ & $\%$ & & & \\
\hline 0 & $\leq 400$ & 45 & 6.00 & 2 & 0.27 & 1.00 & - & - \\
\hline 1 & $\leq 400$ & 54 & 7.20 & 16 & 2.13 & 0.22 & $0.05-1.00$ & 0.07 \\
\hline 2 & $\leq 400$ & 45 & 6.00 & 23 & 3.07 & 0.38 & $0.08-1.86$ & 0.23 \\
\hline 3 & $\leq 400$ & 33 & 4.40 & 44 & 5.87 & 1.00 & $1.00-0.21$ & 1.00 \\
\hline 4 & $\leq 400$ & 23 & 3.07 & 32 & 4.27 & 1.04 & $0.21-5.12$ & 0.96 \\
\hline 5 & $\leq 400$ & 2 & 0.27 & 3 & 0.40 & 1.12 & $0.11-11.60$ & 0.92 \\
\hline 0 & 400 & 3 & 0.40 & 4 & 0.53 & 1.82 & $0.51-2.85$ & 0.49 \\
\hline 1 & 400 & 45 & 6.00 & 24 & 3.20 & 1.80 & $0.85-3.80$ & 0.12 \\
\hline 2 & 400 & 28 & 3.73 & 123 & 16.40 & 8.60 & $4.50-16.44$ & $<0.01$ \\
\hline 3 & 400 & 11 & 1.47 & 153 & 20.40 & 10.43 & $4.88-22.31$ & $<0.01$ \\
\hline 4 & 400 & 9 & 1.20 & 57 & 7.60 & 4.55 & $1.88-11.02$ & $<0.01$ \\
\hline 5 & 400 & 1 & 0.13 & 4 & 0.53 & 2.67 & $0.16-45.14$ & 0.50 \\
\hline
\end{tabular}

OR: odds ratio; $95 \%$ CI: $95 \%$ confidence interval.

Table 6. Combined analysis of drinking status, and variant genotype distribution in relation to oral squamous cell carcinoma susceptibility.

\begin{tabular}{|c|c|c|c|c|c|c|c|c|}
\hline \multirow{2}{*}{$\begin{array}{c}\text { Number of } \\
\text { Genotypes }\end{array}$} & \multirow{2}{*}{$\begin{array}{c}\text { Drinking } \\
\text { Status }\end{array}$} & \multicolumn{2}{|c|}{ Control Group } & \multicolumn{2}{|c|}{ Patient Group } & OR Value & 95\% CI & $p$ Value \\
\cline { 3 - 9 } & $n$ & $\%$ & $n$ & $\%$ & & & \\
\hline 0 & {$[-]$} & 100 & 13.33 & 27 & 3.60 & 1.00 & - & - \\
\hline 1 & {$[-]$} & 166 & 22.13 & 42 & 5.60 & 0.41 & $0.21-0.80$ & 0.01 \\
\hline 2 & {$[-]$} & 105 & 14.00 & 77 & 10.27 & 1.18 & $0.61-2.28$ & 0.62 \\
\hline 3 & {$[-]$} & 51 & 6.80 & 65 & 8.67 & 2.05 & $1.03-4.11$ & 0.04 \\
\hline 4 & {$[-]$} & 43 & 5.73 & 43 & 5.73 & 1.61 & $0.78-3.32$ & 0.20 \\
\hline 5 & {$[-]$} & 3 & 0.40 & 2 & 0.27 & 1.07 & $0.16-7.06$ & 0.94 \\
\hline 0 & {$[+]$} & 29 & 3.87 & 18 & 2.40 & 0.44 & $0.21-0.90$ & 0.03 \\
\hline 1 & {$[+]$} & 52 & 6.93 & 93 & 12.40 & 7.07 & $4.38-11.42$ & $<0.01$ \\
\hline 2 & {$[+]$} & 89 & 11.87 & 160 & 21.33 & 2.45 & $1.66-3.63$ & $<0.01$ \\
\hline 3 & {$[+]$} & 55 & 7.33 & 116 & 15.47 & 1.66 & $1.02-2.69$ & 0.04 \\
\hline 4 & {$[+]$} & 41 & 5.47 & 84 & 11.20 & 2.05 & $1.67-3.60$ & 0.01 \\
\hline 5 & {$[+]$} & 16 & 2.13 & 23 & 3.07 & 2.16 & $0.32-14.41$ & 0.43 \\
\hline
\end{tabular}

OR: odds ratio; 95\% CI: 95\% confidence interval. 
Table 7. Combined analysis of drinking index, and variant genotype distribution in relation to oral squamous cell carcinoma susceptibility.

\begin{tabular}{|c|c|c|c|c|c|c|c|c|}
\hline \multirow{2}{*}{$\begin{array}{c}\text { Number of } \\
\text { Genotypes }\end{array}$} & \multirow{2}{*}{$\begin{array}{c}\text { Drinking } \\
\text { Index }\end{array}$} & \multicolumn{2}{|c|}{ Control Group } & \multicolumn{2}{|c|}{ Patient Group } & OR Value & 95\% CI & $p$ Value \\
\hline & $n$ & $\%$ & $n$ & $\%$ & & & \\
\hline 0 & $\leq 3000$ & 21 & 2.80 & 3 & 0.40 & 1.00 & - & - \\
\hline 1 & $\leq 3000$ & 47 & 6.27 & 15 & 2.00 & 0.80 & $0.01-077$ & 0.03 \\
\hline 2 & $\leq 3000$ & 49 & 6.53 & 26 & 3.47 & 0.13 & $0.01-1.25$ & 0.08 \\
\hline 3 & $\leq 3000$ & 36 & 4.80 & 38 & 5.07 & 0.26 & $0.03-2.47$ & 3.79 \\
\hline 4 & $\leq 3000$ & 16 & 2.13 & 28 & 3.73 & 0.44 & $0.05-4.26$ & 0.48 \\
\hline 5 & $\leq 3000$ & 3 & 0.40 & 4 & 0.53 & 0.33 & $0.02-4.74$ & 0.42 \\
\hline 0 & 3000 & 1 & 0.13 & 4 & 0.53 & 2.96 & $0.55-6.28$ & 0.08 \\
\hline 1 & 3000 & 24 & 3.20 & 44 & 5.87 & 5.74 & $2.67-12.35$ & $<0.01$ \\
\hline 2 & 3000 & 39 & 5.20 & 122 & 16.27 & 5.90 & $3.27-10.71$ & $<0.01$ \\
\hline 3 & 3000 & 30 & 4.00 & 138 & 18.40 & 4.36 & $2.38-7.97$ & $<0.01$ \\
\hline 4 & 3000 & 14 & 1.87 & 54 & 7.20 & 2.20 & $0.94-5.16$ & 0.07 \\
\hline 5 & 3000 & 2 & 0.27 & 18 & 2.40 & 6.75 & $0.83-54.66$ & 0.07 \\
\hline
\end{tabular}

OR: odds ratio; $95 \%$ CI: $95 \%$ confidence interval.

Table 8. Combined analysis of smoking and drinking status, and variant genotype distribution in relation to oral squamous cell carcinoma susceptibility.

\begin{tabular}{|c|c|c|c|c|c|c|c|c|c|}
\hline \multirow{2}{*}{$\begin{array}{l}\text { Number of } \\
\text { Genotypes }\end{array}$} & \multirow{2}{*}{$\begin{array}{c}\text { Smoking } \\
\text { Status }\end{array}$} & \multirow{2}{*}{$\begin{array}{c}\text { Drinking } \\
\text { Status }\end{array}$} & \multicolumn{2}{|c|}{ Controls } & \multicolumn{2}{|c|}{ Patients } & \multirow{2}{*}{$\begin{array}{c}\text { OR } \\
\text { Value }\end{array}$} & \multirow[t]{2}{*}{$95 \%$ CI } & \multirow[t]{2}{*}{$p$ Value } \\
\hline & & & $n$ & $\%$ & $n$ & $\%$ & & & \\
\hline 0 & {$[-]$} & {$[-]$} & 68 & 9.07 & 15 & 2.00 & 1.00 & - & - \\
\hline 1 & {$[-]$} & {$[-]$} & 82 & 10.93 & 12 & 1.60 & 1.04 & 0.64-1.70 & 0.86 \\
\hline 2 & {$[-]$} & {$[-]$} & 48 & 6.40 & 42 & 5.60 & 2.22 & $1.44-3.44$ & $<0.01$ \\
\hline 3 & {$[-]$} & {$[-]$} & 27 & 3.60 & 21 & 2.80 & 2.14 & $1.31-3.50$ & $<0.01$ \\
\hline 4 & {$[-]$} & {$[-]$} & 18 & 2.40 & 18 & 2.40 & 3.30 & $1.91-5.67$ & $<0.01$ \\
\hline 5 & {$[-]$} & {$[-]$} & 1 & 0.13 & 1 & 0.13 & 0.41 & $0.09-1.87$ & 0.25 \\
\hline 0 & {$[-]$} & {$[+]$} & 24 & 3.20 & 16 & 2.13 & 1.91 & $0.31-11.81$ & 0.48 \\
\hline 1 & {$[-]$} & {$[+]$} & 36 & 4.80 & 31 & 4.13 & 7.07 & $4.38-11.42$ & $<0.01$ \\
\hline 2 & {$[-]$} & {$[+]$} & 68 & 9.07 & 48 & 6.40 & 2.45 & $1.66-3.63$ & $<0.01$ \\
\hline 3 & {$[-]$} & {$[+]$} & 44 & 5.87 & 32 & 4.27 & 1.66 & $1.02-2.69$ & 0.04 \\
\hline 4 & {$[-]$} & {$[+]$} & 22 & 2.93 & 28 & 3.73 & 2.05 & $1.17-3.60$ & 0.01 \\
\hline 5 & {$[-]$} & {$[+]$} & 13 & 1.73 & 1 & 0.13 & 2.16 & $0.32-14.40$ & 0.43 \\
\hline 0 & {$[+]$} & {$[-]$} & 32 & 4.27 & 12 & 1.60 & 0.86 & $0.16-4.63$ & 0.87 \\
\hline 1 & {$[+]$} & {$[-]$} & 84 & 11.20 & 30 & 4.00 & 2.53 & $1.51-3.96$ & $<0.01$ \\
\hline 2 & {$[+]$} & {$[-]$} & 57 & 7.60 & 35 & 4.67 & 2.43 & $1.65-3.58$ & $<0.01$ \\
\hline 3 & {$[+]$} & {$[-]$} & 24 & 3.20 & 44 & 5.87 & 4.90 & $2.92-8.21$ & $<0.01$ \\
\hline 4 & {$[+]$} & {$[-]$} & 25 & 3.33 & 25 & 3.33 & 1.60 & $0.91-2.80$ & 0.10 \\
\hline 5 & {$[+]$} & {$[-]$} & 2 & 0.27 & 1 & 0.13 & 32.20 & 5.49-188.91 & $<0.01$ \\
\hline 0 & {$[+]$} & {$[+]$} & 5 & 0.67 & 2 & 0.27 & 3.45 & $1.62-5.41$ & $<0.01$ \\
\hline 1 & {$[+]$} & {$[+]$} & 16 & 2.13 & 62 & 8.27 & 10.36 & $6.53-16.44$ & $<0.01$ \\
\hline 2 & {$[+]$} & {$[+]$} & 21 & 2.80 & 112 & 14.93 & 6.20 & $4.12-9.33$ & $<0.01$ \\
\hline 3 & {$[+]$} & {$[+]$} & 11 & 1.47 & 84 & 11.20 & 5.23 & $3.33-820$ & $<0.01$ \\
\hline 4 & {$[+]$} & {$[+]$} & 19 & 2.53 & 56 & 7.47 & 4.19 & $2.67-6.58$ & $<0.01$ \\
\hline 5 & {$[+]$} & {$[+]$} & 3 & 0.40 & 22 & 2.93 & 13.26 & $4.09-42.97$ & $<0.01$ \\
\hline
\end{tabular}

OR: odds ratio; $95 \% \mathrm{CI}$ : 95\% confidence interval. 
Smokers with five variant genotypes were 32-times more likely to have OSCC.

Furthermore, within the sub-population of smokers, SI was synergistic with multiple variant genotypes (Table $5)$. In the patient group, the presence of multiple variant genotypes and an SI $>400$ was significantly more common compared to the control group $(p<0.01)$.

Drinking Status and Gene Polymorphisms. A similar analysis was applied to CYP1A1-MspI (m2/m2), EC-SOD (C/G), GSTT1 [-], GSTM1 [-] and ALHD2 (non $\mathrm{G} / \mathrm{G}$ ) variant genotypes and alcohol consumption (Table 6). The presence of multiple variants and alcohol consumption was significantly more common in patients compared to the control group; furthermore, as with smoking, the drinking behavior and combined variant genotypes had a synergistic effect on the likelihood of cancer.

The synergistic effect of the DI and combined expression of variant genotypes is depicted in Table 7. In the patient group, the presence of multiple variants and a DI $>3000$ was significantly more common compared to the control group $(p<0.01)$.

Analysis of Combined Smoking/Drinking Status and Gene Polymorphisms. Table 8 displays the results of the analysis of the presence of variant genotypes combined with smoking and drinking status. In the patient group, there were significantly more patients who had multiple variants and were positive for both smoking and drinking history as compared to the control group.

\section{DISCUSSION}

The pathogenesis of OSCC is complex, involving combined action of a variety of environmental and genetic factors [11-13]. Smoking and drinking are the main risk factors for OSCC $[14,15]$. Our findings confirm that smoking and drinking were significantly more frequent in individuals with OSCC than in the control individuals. Heavy cigarette and alcohol consumption were also significantly higher in the OSCC group compared to the control group.

The CYP1A1 is a member of the cytochrome P450 family involved in the metabolism of exogenous materials, encoding aryl hydrocarbon hydrolase (AHH), and activating polycyclic aromatic hydrocarbon and aromatic amine [16]. Our results showed that the distribution of genotypes for CYP1A1-MspI was significantly different between the patient group and the control group, and that the CYP1A1MspI (m2/m2) genotype may increase susceptibility to OSCC. This finding, aligned with prior studies, shed light on the involvement of the CYP1A1-MspI polymorphism in carcinogenesis through modified enzymatic activity [16]. Our results also confirmed the results of a meta-analysis which found that CYP1A1-MspI increased the odds of OSCC, specifically among Asian populations [30].

Superoxide dismutase is generally considered as the first line antioxidative defense in the body [17]. This enzyme can be highly effective against ROS to protect the cells and tissues from oxidative stress [18]. The SOD dysregulation is correlated with growth of human malignant tumors $[19,20]$. Further, EC-SOD appears to be important for tumor formation [21-23], and is correlated with OSCC [24]. Our study mirrored the earlier studies that indicated EC-SOD association with OSCC and showed a significant difference in distribution of EC-SOD genotypes between the patient and control groups [24].

The GST polymorphisms are also correlated with cancer susceptibility. Glutathione S-transferase can catalyze the binding of electrophilic carcinogens and glutathione, to metabolize compounds that are easily soluble in water and excrete them. GSTM1/GSTT1 has been previously associated with susceptibility to oral carcinomas [25,31]. Our study confirmed that GSTM1[-]/GSTT1[-] genotypes are correlated with susceptibility to OSCC.

Animal studies have found that the in vivo metabolite of ethanol, acetaldehyde, has significant carcinogenic effects [26,27], and ALDH2 is the main enzyme to metabolize acetaldehyde in the liver. Furthermore, $A L D H 2$ gene polymorphism is correlated with a variety of tumors [28-30,32]. Our study confirmed the correlation between $A L D H 2$ (non $\mathrm{G} / \mathrm{G})$ genotypes and increased susceptibility to OSCC.

Interestingly, our study also found significant differences in the prevalence of combined CYP1A1-MspI (m2/ m2), EC-SOD (C/G), GSTT1 [-], GSTM1 [-] and ALHD2 (non $\mathrm{G} / \mathrm{G}$ ) variant genotypes between the patient and control groups. In the patient group, the percentage of patients with a combination of variant genotypes (more than two genotypes) was significantly higher than that of the control group; the relative risks of OSCC in such patients were significantly increased. Previous studies have shown that OSCC patients are more likely to carry multiple variants and have a history of smoking or drinking; the interaction of two or more of these factors enhances risk $[3,5,29,30,32]$. Our simultaneous analyses confirmed these previous results, as individuals with both multiple variant genotypes and a smoking and drinking history exhibited a significantly higher risk of OSCC. Therefore, OSCC risk increases with the increasing amount and period of smoking and drinking. 
Further investigations and studies of the effects of these gene and environmental interactions is paramount to an earlier diagnosis of OSCC. More studies of non Asian populations is another avenue of research worth undertaking.

\section{ACKNOWLEDGMENTS}

This work was supported by Natural Science Foundation of Heilongjiang Province (H201644).

Declaration of Interest. The authors report no conflicts of interest. The authors alone are responsible for the content and writing of this article.

\section{REFERENCES}

1. Gollin SM. Cytogenetic alterations and their molecular genetic correlates in head and neck squamous cell carcinoma: a next generation window to the biology of disease. Genes Chromosomes Cancer. 2014; 53(12):972-990.

2. Warnakulasuriya S. Global epidemiology of oral and oropharyngeal cancer. Oral Oncol. 2009; 45(4-5): 309-316.

3. Bolt WJ, McLaughlin JK, Winn DM, Austin DF, Greenberg RS, Preston-Martin S, et al. Smoking and drinking in relation to oral and pharyngeal cancer. Cancer Res. 1988; 48(11): 3282-3287.

4. Negri E, La Vecchia C, Franceschi S, Tavani A. Attributable risk for oral cancer in northern Italy. Cancer Epidemiol Biomarkers Prev. 1993; 2(3): 189-193.

5. Brunotto M, Zarate AM, Bono A, Barra JL, Berra S. Risk genes in head and neck cancer: A systematic review and meta-analysis of last 5 years. Oral Oncol. 2014; 50(3): 178-188.

6. Kaminagakura E, Caris A, Coutinho-Camillo C, Soares FA, Takahama-Júnior A, Kowalski LP. Protein expression of CYP1A1, CYP1B1, ALDH1A1, and ALDH2 in young patients with oral squamous cell carcinoma. Int J Oral Maxillofac Surg. 2016; 45(6): 706-712.

7. Young RP, Hopkins R, Black PN, Eddy C, Wu L, Gamble GD, et al. Functional variants of antioxidant genes in smokers with COPD and in those normal lung function. Thorax. 2006; 61(5): 394-399.

8. Wilson MH, Grant PJ, Hardie LJ, Wild CP. Glutathione S-transferaseM1 null genotype is associated with a decreased risk of myocardial infarction. FASEB J. 2000; 14(5): 791-796.
9. Nguyen TV, Janssen MJ, Vanoijen MG, Bergevoet $\mathrm{SM}$, te Morsche R, van Asten H, et al. Genetic polymorphisms in GSTA1, GSTP1, GSTT1, and GSTM1 and gastric cancer risk in a Vietnamese population. Oncol Res. 2010; 18(7): 349-355.

10. Ishibashi T, Taguchi A, Yamamoto Y, Harada S. Evaluation of the use of self-reported facial flushing and ethanol patchtest for ALDH2 genotypes. Nihon Arukoru YakubutsuIgakkai Zasshi. 2010; 45(5): 464-476.

11. Hillbertz NS, Hirsch JM, Jalouli J, Jalouli MM, Sand L. Viral and molecular aspects of oral cancer. Anticancer Res. 2012; 32(10): 4201-4212.

12. Bagan J, Sarrion G, Jimenez Y. Oral cancer: Clinical features. Oral Oncol. 2010; 46(3): 414-417.

13. Murugan AK, Munirajan AK, Tsuchida N. Ras oncogenes in oral cancer: The past 20 years. Oral Oncol. 2012; 48(5): 383-392.

14. Lissowska J, Pilarska A, Pilarski P, Samolczyk-Wanyura D, Piekarczyk J, Bardin-Mikolłajczak A, et al. Smoking, alcohol, diet, dentition and sexual practices in the epidemiology of oral cancer in Poland. Eur J Cancer Prev. 2003; 12(1): 25-33.

15. Petersen PE. Oral cancer prevention and control the approach of the World Health Organization. Oral Oncol. 2009; 45(4-5): 454-460.

16. Bartsch H, Nair U, Risch A, Rojas M, Wikman H, Alexandrov K. Polymorphism of CYP genes, alone or in combination, as a risk modifier of tobacco-related cancers. Cancer Epidemiol Biomarkers Prev. 2000; 9(1): 3-28.

17. Blokhina O, Virolainen E, Fagerstedt KV. Antioxidants, oxidative damage and oxygen deprivation stress: A review. Ann Bot. 2003; 91(2): 179-194.

18. Liochev SI, Fridovich I. The effects of superoxide dismutase on $\mathrm{H} 2 \mathrm{O} 2$ formation. Free Radic Biol Med. 2007; 42(10): 1465-1469.

19. Hileman EA, Achanta G, Huang P. Superoxide dismutase: An emerging target for cancer therapeutics. Expert Opin Ther Targets. 2001; 5(6): 697-710.

20. Kinnula VL, Crapo JD. Superoxide dismutases in malignant cells and human tumors. Free Radic Biol Med. 2004; 36(6): 718-744.

21. Moriarty-Craige SE, Jones DP. Extracellular thiols and thiol/disulfide redox in metabolism. Annu Rev Nutr. 2004; 24(4): 481-509.

22. Nakamura H, Masutani H, Yodoi J. Extracellular thioredoxin and thioredoxin-binding protein 2 in control of cancer. Semin Cancer Biol. 2006; 16(6): 444-451. 
23. Rees MD, Kennett EC, Whitelock JM, Davies MJ. Oxidative damage to extracellular matrix and its role in human pathologies. Free Radic Biol Med. 2008; 44(12): 197-2001.

24. Yokoe H, Nomura H, Yamano Y, Fushimi K, Sakamoto Y, Ogawara K, et al. Alteration of extracellular superoxide dismutase expression is associated with an aggressive phenotype of oral squamous-cell carcinoma. Exp Ther Med. 2010; 1(4): 585-590.

25. Pal M, Chaudhuri SR, Jadav A, Banerjee S, Paul RR, Dutta PK, et al. Quantitative dimensions of histopathological attributes and status of GSTM1-GSTT1 in oral submucous fibrosis. Tissue Cell. 2008; 40(6): 425-435.

26. Yokoyama A, Omori T, Yokoyama T. Alcohol and aldehyde dehydrogenase polymorphisms and a new strategy for prevention and screening for cancer in the upper aerodigestive tract in East Asians. Keio J Med. 2010; 59(4): 115-130.

27. Salaspuro M. Acetaldehyde and gastric cancer. J Dig Dis. 2011; 12(2): 51-59.

28. Oze I, Matsuo K, Hosono S, Ito H, Kawase T, Watanabe $\mathrm{M}$, et al. Comparison between self-reported facial flushing after alcohol consumption and ALDH2 Glu504Lys polymorphism for risk of upper aerodigestive tract cancer in a Japanese population. Cancer Sci. 2010; 101(8): 1875-1880.

29. Druesne-Pecollo N, Tehard B, Mallet Y, Gerber M, Norat T, Hercberg S, et al. Alcohol and genetic polymorphisms: Effect on risk of alcohol-related cancer. Lancet Oncol. 2009; 10(2): 173-180.

30. Xie S, Luo C, Shan X, Zhao S, He J, Cai Z. CYP1A1 MspI polymorphism and the risk of oral squamous cell carcinoma: Evidence from a meta-analysis. Mol Clin Oncol. 2016; 4(4): 660-666.

31. Yokoe H, Nomura H, Yamano Y, Fushimi K, Sakamoto Y, Ogawara K, et al. Alteration of extracellular superoxide dismutase expression is associated with an aggressive phenotype of oral squamous-cell carcinoma. Exp Ther Med. 2010; 1(4): 585-590.

32. Tanaka F1, Yamamoto K, Suzuki S, Inoue H, Tsurumaru M, Kajiyama Y, et al. Strong interaction between the effects of alcohol consumption and smoking on oesophageal squamous cell carcinoma among individuals with ADH1B and/or ALDH2 risk alleles. Br Med J. 2010; 59 (11): 1457-64. 
